\title{
The comparison of standard and tubeless percutaneous nephrolithotomy procedures
}

Ismail Nalbant, Ufuk Ozturk, Nevzat Can Sener, Onur Dede, Ahmet Murat Bayraktar, M. Abdurrahim Imamoglu

Department of Urology, Ministry of Health, Yenimahalle State Hospital (IN, AMB); Department of Urology, Ministry of Health, Ankara Dıskapı Yıldırım Beyazit Education and Research Hospital (UO, NCS, MAI) and Department of Urology Ministry of Health, Gölbasi Hasvak State Hospital (OD), Ankara, Turkey

ABSTRACT

Purpose: To compare totally tubeless and standard percutaneous nephrolitotomy procedures on many parameters.

Materials and Methods: Percutaneous nephrolitotomy was performed on 195 patients between June 2009 and May 2012. The data of those patients were evaluated retrospectively. Totally tubeless cases were enrolled to Group 1, and Group 2 consisted of non-tubeless cases (re-entry or Foley catheter).

Results: Group 1 included 85 cases and group 2 a total of 110 patients. Paper tracing values for the kidney stones were $321.25 \pm 102.4 \mathrm{~mm}^{2}$ and $324.10 \pm 169.5 \mathrm{~mm}^{2}$ respectively. Mean fluoroscopy time was $4.9 \pm 1.9 \mathrm{~min}$ and $5.08 \pm 2.7 \mathrm{~min}$, mean operation time was $78.8 \pm 27.9 \mathrm{~min}$ and $81.9 \pm 28.77 \mathrm{~min}$ and mean decrease in hematocrit was $2.6 \pm 1.6$ and $3.74 \pm 1.9$ respectively. All these comparisons were statistically significant. Length of hospitalization was $1.6 \pm 1.1$ and $3.5 \pm 1.5$ days for Groups 1 and 2 respectively. Mean superficial pain score was $5.8 \pm 1.6$ and $6.7 \pm 1.2$ respectively for both groups after 1 hour. At 6 hours, the scores changed to $3.87 \pm 1.22$ and $4.84 \pm 1.3$ respectively. The analgesic dose was $1.00 \pm 0.7$ and $1.53 \pm 0.6$ for the groups respectively at 6 hours. All the statistical differences were significant for these three parameters. Conclusions: We believe that, because of their post operative patient comfort and decreased length of hospital stay, totally tubeless procedures should be considered as an alternative to standard percutaneous nephrolitotomy.

\section{ARTICLE INFO}

\section{Key words:}

Kidney Calculi; Nephrostomy,

Percutaneous; percutaneous

nephrolitotomy; Kidney

Tubules, Distal

Int Braz J Urol. 2012; 38: 795-801

Submitted for publication:

June 05, 2012

Accepted after revision:

October 10, 2012

\section{INTRODUCTION}

Kidney stones have been a frequent, significantly morbid problem. Patients need to undergo invasive surgery and to go through a hard time with long recovery. Until the last 2 decades, open surgery for kidney stones was a must. Because of the morbidity of those operations, new modalities were researched and Percutaneous Nephrolitotomy (PCNL) was described as a treatment method.
Initially, Rupel and Brown's removal of the obstructing kidney stone (1), Fenstrom and Johansson published their report on the new stone surgery, which they called Percutaneous Pyelolithotomy (2). The method quickly gained popularity and all patients assigned for open surgery were told to be candidates for percutaneous surgery. After gaining experience with the standard method, surgeons tended to perform this operation with even more decreased morbidity. To achieve that goal, 
they got rid of the nephrostomy tube and tubeless PCNL emerged.

We, in this study, aimed to compare the results of the traditional PCNL with the less invasive method, tubeless PCNL, retrospectively.

\section{MATERIALS AND METHODS}

Data of 195 patients submitted to PCNL in our clinic between June 2009 and May 2012 were evaluated retrospectively. Two groups were formed: Group 1 included patients submitted to totally tubeless and Group 2 to standard PCNL (with any nephrostomy tube). All procedures were performed by a single surgeon. The groups had no differences between operation technique- including dilatation size and type. Pre and post operative hematocrit values, blood transfusion rates, fluoroscopy durations, post operative complications, length of hospital stay, post operative $1^{\text {st }}$ and $6^{\text {th }}$ hours pain scores and analgesic requirements were compared in tubeless and standard patients. Patients having staghorn calculi, requiring multiple accesses were excluded from the study. Pain scores were performed using the Visual Analog Scale. In group 2, patients were checked for residual fragments by X-ray and in addition to this, renal ultrasound was performed to patients in Group 1 for urinoma, hematoma or clinically significant residual fragments.

Inclusion criteria for the totally tubeless PCNL during the operation were intact collecting system (no perforations), no serious bleeding, having one access, no previous operations or drai- nage and no serious extravasations determined by retrograde pyelogram at the end of the operation. Achieving stone free patients or patients having clinically insignificant residual fragments (CIRF) (smaller than 4 millimeters,-mm-), no bleeding for 5 minutes after finalization of operation, were also considered as inclusion criteria.

All patients had been evaluated with complete blood count, plasma electrolytes, kidney and liver function tests, coagulation parameters and urine analysis and urine cultures. Patients with urinary tract infections (UTI) were treated with antibiotics and operation was performed after sterile urine cultures.

Measuring the largest edge and the edge perpendicular and multiplying them calculated the stone load. With multiple stones, the measurement was done to all stones and added.

Statistical analysis was done using SPSS for Windows 15.0 and Mann Withney $\mathrm{U}$ test. P values $<0.05$ were considered statistically significant.

\section{RESULTS}

We evaluated the data of 195 cases undergoing PCNL in our clinic. There were 85 patients in Group 1 and 110 in group 2. The groups had similar demographics according to co-morbidities. They are summarized in Table-1. Mean stone burden was $321.25 \pm 102.4 \mathrm{~mm}^{2}$ in Group 1 and, $324.10 \pm 169.5 \mathrm{~mm}^{2}$ in Group 2. Even though mean stone load, fluoroscopy duration and operative time was higher in Group 2, the difference was not statistically significant.

Table 1 - Co-morbidites and BMI of both groups.

\begin{tabular}{|c|c|c|c|}
\hline & Group $1(n=85)$ & Group $2(n=110)$ & $\mathrm{p}$ \\
\hline Hypertension & $10(11.7 \%)$ & $14(12.7 \%)$ & $>0.05$ \\
\hline Diabetes Mellitus & $7(8 \%)$ & $10(9 \%)$ & $>0.05$ \\
\hline Chronic Respiratory Diseases & $4(4.7 \%)$ & $6(5.4 \%)$ & $>0.05$ \\
\hline BMI (Mean \pm SD) & $23.6 \pm 0.18$ & $24.1 \pm 0.27$ & $>0.05$ \\
\hline
\end{tabular}


Need for analgesics and VAS scores at $1^{\text {st }}$ and $6^{\text {th }}$ hours were statistically lower for patients in Group 1. Parameters concerning the operation are summarized in Table-2.

In Group 1, for 78 (91.6\%) patients and in Group 2, for 96 (87.7\%) patients complete stone removal was achieved. 7 (8.2\%) cases in Group 1 had prolonged (at least 24 hours) leakage. Six patients were treated by placing a double $\mathrm{j}$ stent, the other patient undergone ureteroscopy for distal ureter stone. In group 2, 11 (10\%) cases had prolonged leakage. 3 of these cases (2.7\%) had to undergo ureterorenoscopy and 7 were treated by group 1 were evaluated by renal ultrasonography and no pathology was revealed. In Group 2, one patient suffered from toxic hepatitis, caused by hyper reaction to anesthesia. Postoperative cultures of 3 patients revealed E.coli, but that was not found to be statistically significant. No major complications were seen in both groups.

\section{COMIMENTS}

The European Association of Urology (EAU) recommends PCNL for kidney stones larger than $2 \mathrm{~cm}$ in its guideline on Urolithiasis (3).

Table 2 - Operative parameters of both groups.

\begin{tabular}{|c|c|c|c|}
\hline & $\begin{array}{l}\text { Group } 1 \\
(n=85)\end{array}$ & $\begin{array}{l}\text { Group } 2 \\
(n=110)\end{array}$ & $p$ \\
\hline $\begin{array}{l}\text { Length of hospitalization (days) } \\
\text { Mean } \pm \text { SD }\end{array}$ & $1.6 \pm 1.1$ & $3.5 \pm 1.5$ & $<0.05$ \\
\hline $\begin{array}{l}\text { Length of fluoroscopy (min.) } \\
\text { Mean } \pm \text { SD }\end{array}$ & $4.9 \pm 1.9$ & $5.08 \pm 2.7$ & $>0.05$ \\
\hline $\begin{array}{l}\text { Hematocrit decrease } \\
\text { Mean } \pm \text { SD }\end{array}$ & $2.6 \pm 1.6$ & $3.74 \pm 1.9$ & $<0.05$ \\
\hline $\begin{array}{l}\text { Operative time (min) } \\
\text { Mean } \pm \text { SD }\end{array}$ & $78.8 \pm 27.9$ & $81.9 \pm 28.7$ & $>0.05$ \\
\hline $\begin{array}{l}\text { VAS score (1st hour) } \\
\text { Mean } \pm \text { SD }\end{array}$ & $5.8 \pm 1.6 / 10$ & $6.7 \pm 1.2 / 10$ & $<0.05$ \\
\hline $\begin{array}{l}\text { VAS score (6th hour) } \\
\text { Mean } \pm \text { SD }\end{array}$ & $3.9 \pm 1.22 / 10$ & $4.8 \pm 1.3 / 10$ & $<0.05$ \\
\hline $\begin{array}{l}\text { Analgesic dose in first } 6 \text { hours } \\
\text { Mean } \pm S D\end{array}$ & $1 \pm 0.7$ & $1.53 \pm 0.6$ & $<0.05$ \\
\hline
\end{tabular}

VAS: Visual Analog Scale

retrograde double $\mathrm{j}$ stenting. There were no complications postoperatively. in Table-3.

Postoperative outcomes are summarized

All patients were evaluated with urinary $\mathrm{X}$-ray post operatively. In addition, all patients in
In our practice, placing a nephrostomy tube after PCNL is the standard method. In the last years, tubeless PCNL, the procedure without placing a nephrostomy tube, neither internally nor externally was described. By placing a nephrostomy tube, the surgeon obtains adequate urinary 
Table 3 - Success rates of both groups.

\begin{tabular}{lccc}
\hline & $\begin{array}{c}\text { Group 1 } \\
(\mathrm{n}=85)\end{array}$ & $\begin{array}{c}\text { Group 2 } \\
(\mathrm{n}=110)\end{array}$ & $p$ \\
\hline Stone free & $78(91.6 \%)$ & $96(87.7 \%)$ & $>0.05$ \\
Clinically insignificant residual fragments & $4(4.1 \%)$ & $6(5.4 \%)$ & $>0.05$ \\
DJ & $7(8.2 \%)$ & $11(10 \%)$ & $>0.05$ \\
URS & $1(1.1 \%)$ & $2(2.7 \%)$ & $>0.05$ \\
Residual fragments & $1(1.1 \%)$ & $3(2.7 \%)$ & $>0.05$ \\
\hline
\end{tabular}

DJ: Double-J ureteral stent.

URS: Ureteroscopy.

discharge, hemostasis, tract recovery and a guide for a second operation, if needed. However, because it can cause pain in early hours, it can deteriorate the patient comfort. In the series published by Bdesha, patients with tubeless PCNL were hospitalized for about 2 days, and there was no need for urgent placing of a tube. Hemedra reported a $1.2 \mathrm{gr} / \mathrm{dL}$ decrease in hemoglobin and suggested the tubeless procedure in patients with solitary kidney. Goh and Wolf reported decreased morbidity with tubeless operations (4).

After the rapid advancement in PCNL, some surgeons have a tendency on not placing the nephrostomy tube (5). Zilberman et al. reviewed the papers about tubeless PCNL. They reported similar results with tubeless PCNL compared to standard PCNL (6). With this approach, the target is to achieve less hospital days, less pain scores, less analgesics, faster return to normal activities and lower costs.

In addition, there are some indications for tubeless PCNL, such as cases with single tracts, no distal obstruction, no intraoperative complications (such as calyx perforation) and not planning the second look $(7,8)$.

In the study designed by Karami et al. (9), 210 patients had undergone tubeless PCNL. All patients had over $2 \mathrm{~cm}$ kidney stones (avg $3 \mathrm{~cm}$ ) and 21 had staghorn stones. 91.04\% of the cases were stone free, and $8.95 \%$ (18 patients) had residual fragments around $7 \mathrm{~mm}$ and they all were treated by SWL. 40 patients had minor bleeding, 22 patients (10.9\%) needed blood transfusion and 16 patients (7.9\%) suffered UTI. For pain management, diclofenac or indomethacin was used; $50 \mathrm{mg}$ of petidine was used for 10 patients. Mean hospitalization time was 3.5 days. The researchers underlined that tubeless PCNL is a safe and economic approach with high patient comfort (9).

In a similar study, Shah et al. (10) randomized patients to tubeless and a small diameter (8F) nephrostomy tube and compared their pain, need for analgesics and days of hospitalization. Tubeless group were placed a 6 F Double J tube. That group had less pain, need for analgesics and days of hospitalization. But $39.4 \%$ of the same group suffered pain from Double J.

Tubeless PCNL indications expanded in recent literature. Jung and Bellman used the technique successfully on obese patients (5). Shuh et al. performed on bilateral kidney stones. Jou et al. underlined the fact that over $3 \mathrm{~cm}$ or staghorn stones were also candidates for tubeless PCNL (11).

PCNL is a challenging operation; even in the most experienced hands around 1.1-83\% complications may emerge. Hemorrhage, cured by intervention (0.6-17\%) is the most important complication $(12,13)$. Bleeding may occur during 
needle entry, tract dilatation, and nephroscopy. Placing a nephrostomy tube usually avoids this complication. This might mean that in tubeless PCNL it is not possible to perform hemostasis. Even though it is said that diathermia can be used for intrarenal hemorrhage and fibrin injection can be used for parenchymal bleeding, these are used only in experimental studies $(14,15)$. Cormio et al. used Tachosil ${ }^{\circledR}$ for bleeding, and reported less hospital stay for tubeless patients (16). We did not use any additional method for hemostasis. de la Rosette et al. published the data of 96 centers and 5803 patients. They reported 7.8\% significant bleeding, 3.4\%, renal pelvis perforation and 1.8\%. hydrothorax; blood transfusion was administered in $328(5.7 \%)$ patients, and fever $>38.5{ }^{\circ} \mathrm{C}$ occurred in $10.5 \%$ of patients (17).

In our study, our data tend to be parallel to current literature. Even though it was not statistically significant, duration of operation and fluoroscopy were both longer in Group 2. It was considered to be because of the extra time spent to place the nephrostomy tube. Performing a tubeless PCNL is decided at the end of the operation. By that means, the higher number of patients with decrease in hematocrit in Group 2 was considered on this matter. Mean hospitalization days were higher in Group 1 ( $p<0.05)$. Also, pain scores and need for analgesics were higher in Group 2 ( $p<0.05)$. All those parameters are about patient comfort and being able to return to everyday activities. We suggest choosing tubeless PCNL when possible.

\section{CONCLUSIONS}

Because of the superiority of PCNL over open surgery about safety, easily usability, being able to gain more stone-free rates and patient comfort, it is commonly used on stone disease. Parallel to that fact, post operative patient comfort, shorter hospitalization, less need for analgesics might make tubeless PCNL the new standard. In suitable cases, it can be used safely as standard PCNL.

Despite our study's low patient population and retrospective nature, for cases without collecting system perforation or intraoperative hemor- rhage, we suggest the use of tubeless PCNL safely. Prospective randomized studies including larger populations are needed to back up these results.

\section{CONFLICT OF INTEREST}

None declared.

\section{REFERENCES}

1. Rupel E, Brown R: Nephroscopy with removal of stone following nephrostomy for obstructive calculus anuria. J Urol. 1941; 46: 177.

2. Fernström I, Johansson B: Percutaneous pyelolithotomy. A new extraction technique. Scand J Urol Nephrol. 1976; 10: 257-9.

3. Turk C, Knoll T, Petrik A, Sarika K, Straub M: Guidelines on urolithiasis. 2012; 40: 362-71.

4. Goh M, Wolf JS Jr: Almost totally tubeless percutaneous nephrolithotomy: further evolution of the technique. J Endourol. 1999; 13: 177-80.

5. Bellman GC, Davidoff R, Candela J, Gerspach J, Kurtz S, Stout L: Tubeless percutaneous renal surgery. J Urol. 1997; 157: 1578-82.

6. Zilberman DE, Lipkin ME, de la Rosette JJ, Ferrandino MN, Mamoulakis C, Laguna MP,et al.: Tubeless percutaneous nephrolithotomy--the new standard of care? J Urol. 2010; 184: 1261-6.

7. Aghamir SM, Hosseini SR, Gooran S: Totally tubeless percutaneous nephrolithotomy. J Endourol. 2004; 18: 647-8.

8. Limb J, Bellman GC: Tubeless percutaneous renal surgery: review of first 112 patients. Urology. 2002; 59: 527-31; discussion 531.

9. Karami H, Jabbari M, Arbab AH: Tubeless percutaneous nephrolithotomy: 5 years of experience in 201 patients. J Endourol. 2007; 21: 1411-3.

10. Shah HN, Sodha HS, Khandkar AA, Kharodawala S, Hegde SS, Bansal MB: A randomized trial evaluating type of nephrostomy drainage after percutaneous nephrolithotomy: small bore v tubeless. J Endourol. 2008; 22: 1433-9.

11. Jou YC, Cheng MC, Lin CT, Chen PC, Shen JH: Nephrostomy tube-free percutaneous nephrolithotomy for patients with large stones and staghorn stones. Urology. 2006; 67: 30-4.

12. Michel MS, Trojan L, Rassweiler JJ: Complications in percutaneous nephrolithotomy. Eur Urol. 2007; 51: 899-906; discussion 906.

13. Skolarikos A, de la Rosette J: Prevention and treatment of complications following percutaneous nephrolithotomy. Curr Opin Urol. 2008; 18: 229-34. 
14. Aron M, Goel R, Kesarwani PK, Gupta NP: Hemostasis in tubeless PNL: point of technique. Urol Int. 2004; 73: 244-7.

15. Noller MW, Baughman SM, Morey AF, Auge BK: Fibrin sealant enables tubeless percutaneous stone surgery. J Urol. 2004; 172: 166-9.

16. Cormio L, Perrone A, Di Fino G, Ruocco N, De Siati M, de la Rosette J, et al.: TachoSil(@) sealed tubeless percutaneous nephrolithotomy to reduce urine leakage and bleeding: outcome of a randomized controlled study. J Urol. 2012; 188: $145-50$.
17. de la Rosette J, Assimos D, Desai M, Gutierrez J, Lingeman J, Scarpa R, et al.: The Clinical Research Office of the Endourological Society Percutaneous Nephrolithotomy Global Study: indications, complications, and outcomes in 5803 patients. J Endourol. 2011; 25: 11-7.

\section{Correspondence address:} Dr. Ufuk Ozturk Department of Urology Ministry of Health Ankara Dıskapı Yıldırım Beyazit Education and Research Hospital Ankara, Turkey

Telephone: + 9031 2596-2243 E-mail: ufukozturk71@gmail.com 


\section{EDITORIAL COMMENT}

Several publications have been done in the last decade pro tubeless PCNL including prospective and randomized trials (1-3). The present study does not represent any news, but have a representative number of patients included. Do not put a tube after PCNL it is necessary that no complication have been occurred, like significant bleeding, perforation

\section{REFERENCES}

1. Chang CH, Wang CJ, Huang SW: Totally tubeless percutaneous nephrolithotomy: a prospective randomized controlled study. Urol Res. 2011; 39: 459-65.

2. Istanbulluoglu MO, Ozturk B, Gonen M, Cicek T, Ozkardes H: Effectiveness of totally tubeless percutaneous nephrolithotomy in selected patients: a prospective randomized study. Int Urol Nephrol. 2009; 41: 541-5. of the pelvis and residual fragments otherwise place a tube is imperative. Residual fragments are better identified by CT scan than ultrasound or X-ray. The present study showed the same findings which others authors have been published before: less residual fragments, less hospitalization and lower pain scores and need of analgesics.
3. Aghamir SM, Hosseini SR, Gooran S: Totally tubeless percutaneous nephrolithotomy. J Endourol. 2004; 18: 647-8.

\footnotetext{
Dr. Francisco José Cardoso Cavalcanti Universidade de Pernambuco Faculdade de Ciências Médicas de Pernambuco. Rua Arnóbio Marques, 310 - Santo Amaro Recife, PE, 50100-130, Brazil E-mail: francisco.cavalcanti@terra.com.br
} 